\title{
Whose research is it?
}

SIR - The letter by Alan Mackay (Nature 362, 489; 1993) on the handing over of copyrights to commercial interests points to a similar and perhaps more disturbing trend - that of effectively giving away the rights in one's research to pharmaceutical companies. Commercial companies are often the only source of compounds required to conduct investigations of physiological and pharmacological phenomena. They are usually willing to provide a 'free sample' of the compound(s), but only on condition that a restrictive agreement is signed, either by the researcher or by the head of his or her institute.

By these means, the investigator effectively transfers his or her rights and ownership of the research carried out to the company, and subjects his or her self to virtually the same conditions as the company's employees. Here are some examples of the wording used by pharmaceuticals companies in such contracts: - Any discovery or invention (whether patentable or not) arising as a result of the studies with the compound(s) shall belong to $\mathrm{XXX}$.

- All test results shall be submitted to XXX prior to informing any third party either verbally or in writing and they may be used further by XXX without restriction.

- At least 30 days prior to making any submission for publication or other public disclosure, I will provide XXX with a copy thereof for review and comment by XXX. - Any proposal for omissions or modifications [to a manuscript] by XXX shall be respected.

In Denmark, the law prevents university employees from entering into a contract that would ". . . agree limitations to the researcher's right to publish results". Agreements with third parties of the type I have described are allowed only when the external party funds the research in its entirety. And, even then, "The rights to research which results from an agreement of collaboration belong to the researcher". I imagine that similar conditions apply in most countries, so was surprised when representatives of two companies told me that most investigators have no qualms about signing the contracts.

This situation is clearly unsatisfactory. I believe a two-tier system of agreements with the companies would be more appropriate, as follows:

- If a compound is to be used on the basis of a pharmacological property already documented for that compound, the investigator should inform the company of the purpose for which it will be used, and should declare that he will so limit its use, which should then be unrestricted.

- The investigation of novel compounds, which requires absolute secrecy, that collaboration with independent investigators should be financed entirely by the company, which will then own the exclusive rights to the research.

\section{C. Lambert}

Institute of Physiology,

University of Aarhus,

DK-8000 Aarhus C, Denmark

\section{Not so easy}

SIR - You reported earlier this year on the setting up of the German-American Academic Council to support projects and exchanges in science (Nature 362, 779; 1993).

I came to the United States six years ago, newly graduated and full of optimism and energy, but I soon became disillusioned. Although my German diploma was officially evaluated as being equivalent to a master's degree, I could not find employment. When I considered working for a $\mathrm{PhD}$, I was told that I would have to take certain undergraduate courses. I eventually got a job because my immediate supervisor is a European, but I was told that the institute does not recognize my degree. Many employers do not recognize foreign degrees below $\mathrm{PhD}$ level.

Now that I have been given the opportunity, I feel that I am making a valuable contribution to research. But I feel bruised and disillusioned, and when an opportunity arises I shall return to my own country, where my education counts for something.

\section{Ute W. Sherman}

UTM.D. Anderson Cancer Center,

Science Park-Research Division,

Smithville, Texas 78745, USA

\section{Singular events}

SIR - I am organizing a new publication called Journal of Failures to Replicate. Individually, I am sure that many of us have experiences of seriously doubting the replicability of reported studies that ultimately find their way into the textbooks. More importantly, on a philosophical level, there are serious implications within our disciplines for the issue of "when does a finding become an established fact?"

I am keen to hear from anyone who is interested in contributing articles or acting as an academic referee. Naturally, contributions should concern fundamental issues within their relative domains.

\section{Derek Scott}

Department of Psychology.

University of Strathclyde,

Turnbull Building,

155 George Street, Glasgow G1 1RD, UK

\section{Neural net effect}

SIR - In his recent review of Daniel Crevier's book The Tumultous History of the Search for Artificial Intelligence (Nature 364, 199; 1993), Igor Aleksander writes:

"Recent work in connectionism has shown that distributing knowledge over interacting elements (artificial neurons) could lead to a storage requirement that grows only with the logarithm of the number of facts that need to be stored. It seems likely that the brain would have evolved to use this neat trick."

Could he refer your readers to the evidence for this astonishing claim?

H. Christopher Longuet-Higgins

University of Sussex,

Centre for Research

on Perception and Cognition,

Laboratory of Experimental Psychology, Brighton BN1 9QG, UK

\section{Rise and fall}

SIR - Regarding the argument ${ }^{1}$ from the unlikelihood of our being lucky enough to be at the beginning of human civilization and so predicting our species' demise, we are not a random sample. We are at the beginning, because only the early civilized people will not immediately spot this flaw in the argument!

\section{A. J. Hardwick}

University of Cambridge,

Department of Physics,

Cavendish Laboratory,

Madingley Road, Cambridge CB3 OHE, UK

SIR - In this note we shall use the Copernican principle ${ }^{1}$ to estimate the life of Conservative government in Britain. Of course, we have to make the (dubious) assumptions that we are at no special position on the time axis and that there exists an appropriate uniform distribution. Then, using the resulting equations (1) and (2) from ref. 1, with $t_{\text {past }}=14$ years, one finds that (at 50 per cent confidence level) current Conservative rule will continue for a period lying between 4.7 and 42 years. For the worried Conservative, one may say, with 95 per cent confidence, that the future period of their preferred mode of rule is not less than 4.3 months. Those less enthusiastic about the present state of political affairs may draw some comfort from the fact that, at the same 95 per cent confidence level, the current Conservative rule will end within the next 546 years.

\section{P. T. Landsberg}

\section{J. N. Dewynne}

C. P. Please

Faculty of Mathematical Studies,

University of Southampton,

Southampton SO9 5NH, UK

1. Gott III, J. R. Nature 363, 315-319 (1993) 